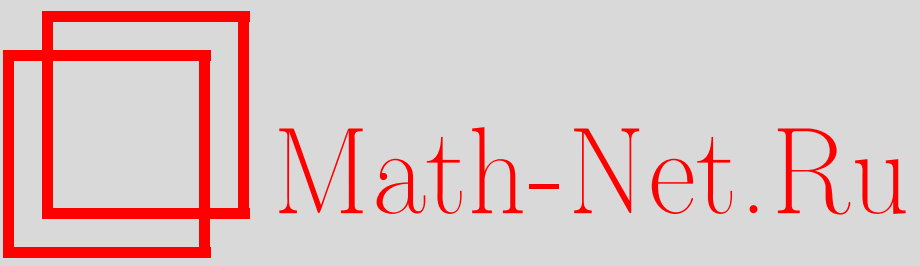

И. А. Чельцов, Трехмерные алгебраические многообразия, обладающие дивизором с численно тривиальным каноническим классом, УМН, 1996, том 51, выпуск $1,177-178$

DOI: https://doi.org/10.4213/rm941

Использование Общероссийского математического портала Math-Net.Ru подразумевает, что вы прочитали и согласны с пользовательским соглашением

http: //www . mathnet.ru/rus/agreement

Параметры загрузки:

IP : 54.237 .59 .107

26 апреля 2023 г., 09:13:06 


\title{
ТРЕХМЕРНЫЕ АЛГЕБРАИЧЕСКИЕ МНОГООБРАЗИЯ, \\ ОБЛАДАЮЩИЕ ДИВИЗОРОМ С ЧИСЛЕННО ТРИВИАЛЬНЫМ КАНОНИЧЕСКИМ КЛАССОМ
}

\author{
И. ЧЕЛЬцов
}

Все многообразия считаются проективными и определенными над $\mathbb{C}$.

Лемма 1. Пусть $\mathscr{G}$ есть рефлексивный пучок на нормальном многообразии $X$ с обильным дивизором $H$. Тогда $H^{i}\left(\mathscr{G} \otimes \mathscr{O}_{X}(-n H)\right)=0$ для $i=0,1$ u $n \gg 0$.

ДоказАТЕльство. Рассмотрим точные последовательности

$$
\begin{gathered}
0 \longrightarrow \mathscr{G} \longrightarrow \mathscr{E} \longrightarrow \mathscr{F} \longrightarrow 0, \\
0 \longrightarrow H^{0}\left(\mathscr{G} \otimes \mathscr{O}_{X}(-n H)\right) \longrightarrow H^{0}\left(\mathscr{E} \otimes \mathscr{O}_{X}(-n H)\right) \longrightarrow H^{0}\left(\mathscr{F} \otimes \mathscr{O}_{X}(-n H)\right) \longrightarrow \cdots,
\end{gathered}
$$

где $\mathscr{E}$ это локально свободный пучок, а $\mathscr{F}$ это пучок без кручения. Легко видеть, что $H^{0}\left(\mathscr{E} \otimes \mathscr{O}_{X}(-n H)\right)=H^{0}\left(\mathscr{F} \otimes \mathscr{O}_{X}(-n H)\right)=0 ; H^{1}\left(\mathscr{E} \otimes \mathscr{O}_{X}(-n H)\right)=0$, поскольку $X$ нормально; (1) влечет $H^{i}\left(\mathscr{G} \otimes \mathscr{O}_{X}(-n H)\right)=0$ для $i=0,1$.

Лемма 2. Пусть нормальное многообразие $X$ содержит обильный дивизор Картье $H$ и дивизор Вейля $D$ такие, что $\operatorname{dim}\{x \in X \mid D$ не дивизор Картье в $x\}=0$ и $\left.D\right|_{Y} \sim 0$ для общего $Y \in|n H|$ при $n \gg 0$. Тогда $D \sim 0$.

ДокАЗАТЕЛЬСтво. Рассмотрим точную последовательность

(2) $H^{0}\left(\mathscr{O}_{X}(D) \otimes \mathscr{O}_{X}(-n H)\right) \longrightarrow H^{0}\left(\mathscr{O}_{X}(D)\right) \longrightarrow H^{0}\left(\mathscr{O}_{Y}\right) \longrightarrow H^{1}\left(\mathscr{O}_{X}(D) \otimes \mathscr{O}_{X}(-n H)\right)$.

$\mathscr{O}_{X}(D)$ реффлексивен (см. $\left.[2,1.6]\right)$ и $H^{i}\left(\mathscr{O}_{X}(D) \otimes \mathscr{O}_{X}(-n H)\right)=0$ для $i=0,1$ и $n \gg 0$ по лемме 1. Из $H^{0}\left(\mathscr{O}_{Y}\right)=\mathbb{C}$ и $(2)$ получаем $H^{0}\left(\mathscr{O}_{X}(D)\right)=\mathbb{C}$, откуда $D \sim 0$.

Теорема. Пусть нормальное трехмерное многообразие $X$ содержит обильный әффективный дивизор Картье $H$ с дювалевскими особенностями и $K_{H} \equiv 0$, тогда $-K_{X} \sim_{\mathbb{Q}} H$ и либо $X$ есть стягивание сечения $\mathbb{P}\left(\mathscr{O}_{H} \oplus \mathscr{O}_{H}\left(\left.H\right|_{H}\right)\right)$, либо $X$ имеет канонические особенности и $H$ это поверхность типа КЗ или поверхность Энриквеса.

ДокаЗАТЕЛЬСТВО. В окрестности $H$ особенности $X$ канонические горенштейновы (см. [3, 7-2-4]) и неканонические особенности $X$ изолированы. Рассмотрим общий элемент $Y \in$ $|n H|$ для $n \gg 0$. Пусть $f: X_{\text {кан }} \rightarrow X$ есть каноническая модификация, $D=12\left(K_{X}+H\right)$, $\widehat{H}=f^{-1}(H), \widehat{Y}=f^{-1}(Y), C=Y \cap H .12 K_{H} \sim 0$ (см. [1]), следовательно $\left.D\right|_{H} \sim 0$, $\left.\left.D\right|_{Y} H\right|_{Y}=\left.\left.D\right|_{H} n H\right|_{H}=D C=0$, но $\left.H\right|_{Y}$ обилен на $Y$. По теореме об индексе $\left.\left.D\right|_{Y} D\right|_{Y}<0$ либо $\left.D\right|_{Y} \equiv 0 ; f$ есть изоморфизм вдоль $H \cup Y$ и

$$
\left.\left.D\right|_{Y} D\right|_{Y}=\left.\left.f^{-1}(D)\right|_{\widehat{Y}} f^{-1}(D)\right|_{\widehat{Y}}=\left.\left.n f^{-1}(D)\right|_{\widehat{H}} f^{-1}(D)\right|_{\widehat{H}}=\left.\left.n D\right|_{H} D\right|_{H}=0 .
$$

Суммируя, получим $\left.D\right|_{Y} \equiv 0,\left.D\right|_{C} \sim 0$. Рассмотрим точную последовательность

$$
0 \longrightarrow H^{0}\left(\mathscr{O}_{Y}\left(\left.D\right|_{Y}\right)\right) \longrightarrow H^{0}\left(\mathscr{O}_{C}\right) \longrightarrow H^{1}\left(\mathscr{O}_{Y}\left(\left.(D-H)\right|_{Y}\right)\right)
$$

По критерию обильности Клеймана $\left.(H-D)\right|_{Y}$ обилен на $Y$ и по теореме об обращении в нуль $($ см. $[3,1-2-5]) H^{1}\left(\mathscr{O}_{Y}\left(\left.(D-H)\right|_{Y}\right)\right)=0$. Из $H^{0}\left(\mathscr{O}_{C}\right)=\mathbb{C}$ и $(3)$ получаем $H^{0}\left(\mathscr{O}_{Y}\left(\left.D\right|_{Y}\right)\right)=\mathbb{C}$ и $\left.D\right|_{Y} \sim 0$. Из леммы 2 следует, что $D=12\left(K_{X}+H\right) \sim 0$.

Предположим, что особенности $X$ не канонические, тогда $K_{X_{\text {кан }}} \sim_{\mathbb{Q}}-\widehat{H}-B$, где $B$ это эффективный не нулевой $\mathbb{Q}$-дивизор (см. $[4,2.18])$. Существует одномерная грань $R \in \mathbf{N E}(X)$

Работа выполнена при поддержке Международного научного фонда (грант № 90000). 
такая, что $-B R<0$. Так как $\widehat{H}$ численно эффективен, то $K_{X_{\text {кан }}} R<0$ и $R$ является экстремальным лучом. Пусть $g: X_{\text {кан }} \rightarrow Z$ есть стягивание $R$, тогда $g$ либо бирационален, либо

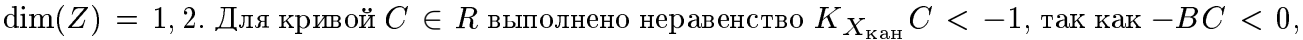
$-\widehat{H} C<0$ и $-\widehat{H} C \in \mathbb{Z}$. Отсюда следует, что $g$ не малое стягивание и не стягивание дивизора на кривую (см. [5, 2.3.2]). Если $g$ есть стягивание дивизора $E$ в точку, то $E$ не лежит в слоях $f$ и эффективный 1-цикл $C=\widehat{Y} \cap E$ содержится в $R$, что противоречит $B C=0$. Если $\operatorname{dim}(Z)=1$, то для любой кривой $l$, лежащей в слое $\left.g\right|_{F}$, где $F$ это исключительный дивизор для $f$, выполнено

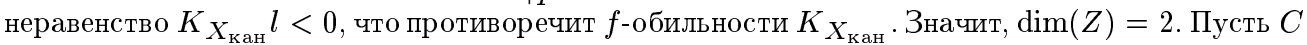
это общий слой $g$, тогда $\widehat{H} C+B C=2, \widehat{H} C \geqslant 1, B C>0$. Откуда $\widehat{H} C=1, B C=1$ и $Z \cong \widehat{H}$. Из $R^{1} g_{*}\left(\mathscr{O}_{X_{\text {кан }}}\right)=0$ получаем точную последовательность

$$
0 \longrightarrow \mathscr{O}_{Z} \longrightarrow g_{*}\left(\mathscr{O}_{X_{\text {кан }}}(\widehat{H})\right) \longrightarrow \mathscr{O}_{\widehat{H}}\left(\left.\widehat{H}\right|_{\widehat{H}}\right) \longrightarrow 0
$$

$g_{*}\left(\mathscr{O}_{X_{\text {кан }}}(\widehat{H})\right)$ есть локально свободный пучок ранга два, $\alpha: g^{*} g_{*} \mathscr{O}_{X_{\text {кан }}}(\widehat{H}) \rightarrow \mathscr{O}_{X_{\text {кан }}}(\widehat{H})$ сюръективен и задает изоморфизм $X_{\text {кан }} \cong \mathbb{P}\left(g_{*} \mathscr{O}_{X_{\text {кан }}}(\widehat{H})\right)$, поскольку $\widehat{H} g$-обилен и является сечением $g$. По теореме об обращении в нуль

$$
\operatorname{Ext}^{1}\left(g_{*}\left(\mathscr{O}_{\widehat{H}}\left(\left.\widehat{H}\right|_{\widehat{H}}\right)\right), \mathscr{O}_{Z}\right)=\operatorname{Ext}^{1}\left(\mathscr{O}_{\widehat{H}}\left(\left.\widehat{H}\right|_{\widehat{H}}\right), \mathscr{O}_{\widehat{H}}\right)=H^{1}\left(\mathscr{O}_{\widehat{H}}\left(-\left.\widehat{H}\right|_{\widehat{H}}\right)\right)=0 .
$$

Следовательно, (4) расщепляется и $X$ есть стягивание сечения $\mathbb{P}\left(\mathscr{O}_{H} \oplus \mathscr{O}_{H}\left(\left.H\right|_{H}\right)\right)$.

Предположим, что особенности $X$ канонические. Нетрудно показать, что в этом случае $H^{1}\left(\mathscr{O}_{X}\right)=H^{2}\left(\mathscr{O}_{X}(-H)\right)=0$. Из точной последовательности

$$
H^{1}\left(\mathscr{O}_{X}\right) \longrightarrow H^{1}\left(\mathscr{O}_{H}\right) \longrightarrow H^{2}\left(\mathscr{O}_{X}(-H)\right)
$$

следует, что $H^{1}\left(\mathscr{O}_{H}\right)=0$ и $H$ есть поверхность типа К3 или поверхность Энриквеса.

\section{СПИСОК ЛИТЕРАТУРЫ}

[1] Beauville A. Surfaces Algebriques Complexes. V. 54. Astérisque. [2] Hartshorne R. // Math. Ann. 1980. V. 254. P. 121-176. [3] Kawamata Y., Matsuda K., Matsuki K. // Adv. Studies in Pure Math. 1987. V. 10. P. 360-383. [4] Kollár J. et al Flips and abundance for algebraic threefolds. Astérisque, 1992. [5] Mori S. // J. AMS. 1988. V. 1. P. 117-253.

Московский государственный

Принято редколлегией университет им. М. В. Ломоносова 01.11 .1995 